PROFESIONALES Y HERRAMIENTAS PARA EL DESARROLLO LOCAL Y SUS SINERGIAS TERRITORIALES. EVALUACIÓN Y PROPUESTAS DE FUTURO IX Coloquio Nacional de Desarrollo Local del GTDL-AGE 

ANTONIO MARTÍNEZ PUCHE, XAVIER AMAT MONTESINOS, ISABEL SANCHO CARBONELL y DANIEL SANCHIZ CASTAÑO (EDS.)

\section{PROFESIONALES Y HERRAMIENTAS PARA EL DESARROLLO LOCAL Y SUS SINERGIAS TERRITORIALES. EVALUACIÓN Y PROPUESTAS DE FUTURO}

IX Coloquio Nacional de Desarrollo Local del GTDL-AGE

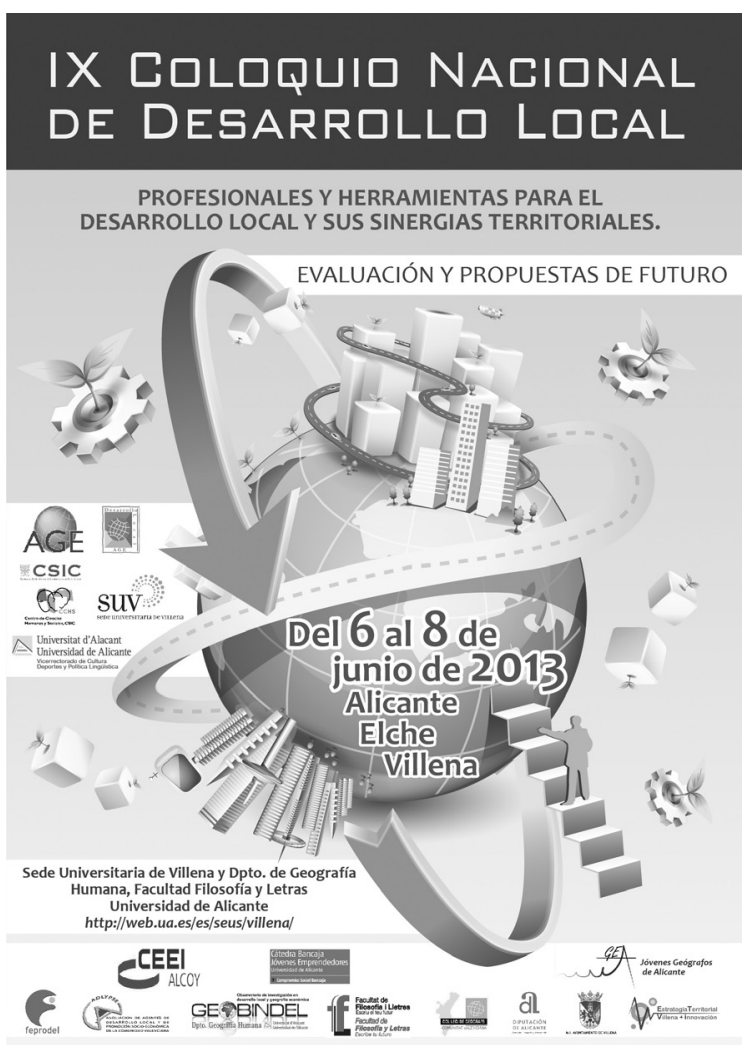


Este libro ha sido examinado y valorado por evaluadores ajenos a la Universidad de Alicante, con el fin de garantizar la calidad científica del mismo.

Publicacions de la Universitat d'Alacant

03690 Sant Vicent del Raspeig

Publicaciones@ua.es

http://publicaciones.ua.es

Telèfon: 965903480

(C) Antonio Martínez Puche, Xavier Amat Montesinos,

Isabel Sancho Carbonell y Daniel Sanchiz Castaño (eds.), 2016

(C) d'aquesta edició: Universitat d'Alacant

ISBN: 978-84-16724-00-0

Dipòsit legal: A 92-2016

Disseny de coberta: candela ink

Composició: Página Maestra (Miguel Ángel Sánchez Hernández)

Impressió i enquadernació: Guada Impresores

\section{unte \\ Unión de Editoriales
Universitarias Españolas \\ WWW.une.es
WWA}

Esta editorial es miembro de la UNE, cosa que garantiza la difusión y comercialización nacional y internacional de sus publicaciones.

Reservados todos los derechos. Cualquier forma de reproducción, distribución, comunicación pública o transformación de esta obra sólo puede ser realizada con la autorización de sus titulares, salvo excepción prevista por la ley. Diríjase a CEDRO (Centro Español de Derechos Repográficos, www.cedro.org) si necesita fotocopias o escanear algún fragmento de esta obra. 


\title{
EL PAPAEL DE LA MUJER EN LA SOCIEDAD SAHARAUI
}

\author{
Hindu Mohamed Ali \\ Estudiante de Doctorado de Desarrollo Local, Facultad de Filosofía y Letras. \\ Universidad de Alicante \\ hindumohamed@gmail.com
}

\section{RESUMEN}

Este trabajo de investigación analiza la importancia de la mujer en la sociedad saharaui actual, partiendo del papel clave y transformador que las mismas tienen en esta sociedad.

El trabajo se basa en la realización de entrevistas tanto a españoles como a los mismos saharauis, con el objeto de confrontar sus opiniones a cerca del papel de las mujeres saharauis en el desarrollo socioeconómico y político. En conclusión, este trabajo pretende destacar el papel de la mujer en la sociedad saharaui.

Palabras clave: Mujeres saharauis, campamentos de refugiados saharauis, sociedad saharaui, opiniones de saharauis y españoles sobre las mujeres saharauis.

\section{THE ROLE OF WOMAN IN THE SAHARAWI SOCIETY}

\section{ABSTRACT}

This research project analyzes the importance of women in Sahrawain society nowdays, starting from the key role and transformations they have in society.

The project is based on interviews both from Spanish and Saharawi women, in order to confront their views about the role of Sahrawi women in the socioeconomical and political development. To conclude, this essay aims to highlight the role of women in Saharawi society.

Keywords: Saharawi women, refugee camps, Saharawi society, Sahrawi opinions and Spanish opinions on Sahrawi women. 


\section{INTRODUCCIÓN}

El propósito de este trabajo es analizar el papel de la mujer en la sociedad saharaui y contextualizar al lector/a sobre la realidad del pueblo saharaui. De este modo, se presentan los hechos históricos más destacados del conflicto saharaui hasta la actualidad, incidiendo en aquéllos que implican el rol activo de la mujer saharaui.

Durante las distintas etapas por las que han pasado las mujeres saharauis, destaca el proceso de emancipación y empoderamiento de la mujer saharaui frente a las mujeres de los países del entorno, la mujer saharaui es libre de tomar sus decisiones. Este logro cabe enmarcarlo en que la sociedad saharaui ha evolucionado a una sociedad musulmana progresista y abierta, donde se separan los derechos de las personas de la religión, según ha expresado uno de los entrevistados con motivo de esta investigación.

Debido a la lucha por la independencia de su país, la mujer saharaui ha avanzado mucho desde la época tribal hasta la actualidad, ya que este factor ha supuesto que la mujer adopte un papel transformador, y que sin ella no puede entenderse ni el progreso ni la organización administrativa de los campamentos de refugiados saharauis. Por este motivo, las mujeres saharauis, gracias a su lucha, están presentes en todos los órganos políticos y administrativos que conforman la República Árabe Saharaui Democrática (RASD).

Actualmente las mujeres siguen su lucha, no sólo para la independencia del Sahara, sino también para garantizar la persistencia de su capacidad de decisión en un Sahara Independiente. Un ejemplo de mujeres que representan a la mujer saharaui son: Aminetu Haidar (líder política y defensora de los derechos de los saharauis) y Marian Hassan, la voz mensajera del pueblo saharaui.

Por todo ello, los esfuerzos que están realizando todos los saharauis, es lo que hace necesario indagar sobre esta cuestión para conocer mejor la realidad del pueblo saharaui, y con una perspectiva de futuro, ante una hipotética resolución del conflicto, debe persistir la participación de las mujeres en los distintos órganos de la República Árabe Saharaui Democrática.

En resumen, con este trabajo se pretende analizar y visibilizar el papel de la mujer saharaui en el esfuerzo para conseguir la construcción de una sociedad en un medio físico donde no se dispone de ningún tipo de recurso y, además, en un entorno geopolítico complejo. Todos los esfuerzos tienen el objetivo de conseguir una estructura política, sanitaria, educativa y social, que permita la mejora de las condiciones de vida de los saharauis. Asimismo, la mujer saharaui es un claro ejemplo que refleja la capacidad de las personas para afrontar nuevos retos; es decir, la capacidad humana de progresar y sobrevivir incluso en condiciones difíciles y complejas. 


\subsection{Justificación}

Este trabajo busca contribuir a que se conozca la evolución de la mujer saharaui a lo largo de distintas etapas y se analiza la situación actual de la mujer respecto a sus derechos y oportunidades frente a los hombres saharauis.

En primer lugar, he decidido hacer este trabajo por motivación personal para sensibilizar a la población acerca del papel fundamental de la mujer saharaui y por motivo académico.

En segundo lugar, en mi opinión resulta de gran interés social llevar a cabo esta investigación debido al escaso conocimiento sobre el papel relevante y clave de las mujeres en la sociedad saharaui pues sin ellas no se entiende el desarrollo de los campamentos de refugiados saharauis.

Por último, con este trabajo quiero hacer un homenaje a todas las mujeres saharauis y reconocerles toda su labor por el progreso de la sociedad saharaui; sin ellas no se entiende el desarrollo humano y social en los campamentos de refugiados saharauis.

\subsection{Objetivos}

El objetivo principal de mi trabajo es comprender el papel de la mujer en la sociedad saharaui, ya que sin ella no se entiende el desarrollo de los Campamentos de Refugiados, y así comprobar su papel clave en la sociedad saharaui. Así mismo los objetivos específicos son los siguientes:

- Analizar la situación de la mujer saharaui en diferentes etapas hasta la actualidad.

- Saber si existe una igualdad de oportunidades tanto de trabajo como de formación entre hombres y mujeres saharauis.

- Observar si existen o no diferencias entre las mujeres que viven en los campamentos de refugiados saharauis y las que residen en España.

\subsection{Metodología}

Para obtener la información necesaria, se ha llevado a cabo un estudio de carácter cualitativo mediante entrevistas semiestructuradas con una serie de preguntas que debían de contestar las personas entrevistadas para confrontar sus opiniones. Con los resultados de las entrevistas se analizó la situación de la mujer dentro de la sociedad saharaui.

Las entrevistas constan de 41 preguntas todas ellas abiertas, destinadas a mujeres y hombres saharauis de distintas edades tanto a los que residen en los campamentos de Refugiados Saharauis como a los saharauis que residen en España. Además, se han realizado entrevistas con 21 preguntas cada una a miembros de distintas asociaciones de toda la geografía española que son conscientes de la realidad del pueblo saharaui y de la lucha de la mujer; estos lazos 
que se han creado entre españoles y saharauis, han sido potenciados con el proyecto Vacaciones en Paz, donde los niños y las niñas saharauis pasan los meses de verano en acogida por parte de familias españolas. Las entrevistas destinas a este colectivo, están compuestas por preguntas similares a las entrevistas realizadas a saharauis. En total se han realizado 46 entrevistas, de las cuales 15 son realizadas a saharauis de ambos sexos residentes en los Campamentos de Refugiados Saharauis, otras 15 realizadas a hombres y mujeres residentes en España y por último 16 entrevistas realizadas a españoles de ambos sexos.

\section{ZONA DE ESTUDIO}

Los Campamentos de Refugiados saharauis, están situados en el sur de Argelia, y en ellos viven aproximadamente 125.000 personas, la mayoría mujeres, niños y ancianos. Los campamentos de refugiados saharauis, han adaptado la estructura administrativa del Sahara Occidental, es decir, los campamentos se dividen en cuatro provincias (wilayas): Auserd, Aaiún, Dajla y Smara.

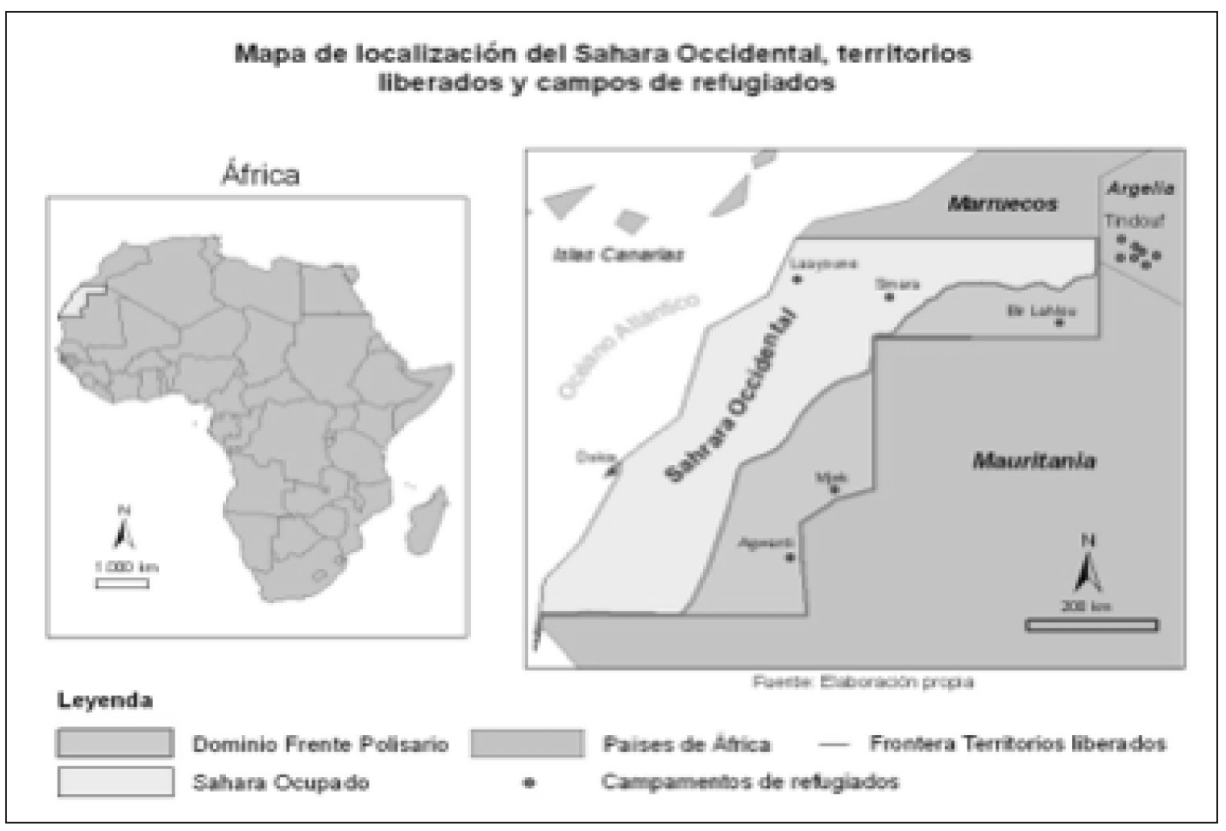

Figura 1: Mapa de localización del Sáhara Occidental, territorios liberados y Campos de Refugiados Saharauis.

Fuente: Elaboración propia. 
Cuadro 1: Habitantes por wilaya ( provincias) año 2010

\begin{tabular}{|c|c|}
\hline Wilayas & Habitantes \\
\hline Aaiún & 34.500 \\
\hline Auserd & 28.800 \\
\hline Smara & 33.200 \\
\hline Dajla & 19.361 \\
\hline 27 de Febrero & 5.500 \\
\hline Total Población & 122.363 \\
\hline
\end{tabular}

Fuente: Entrevistado saharaui a través de la Media Luna Roja Saharaui.

En el cuadro 1, se reflejan los habitantes de cada provincia de los Campamentos de Refugiados Saharauis, no se han contabilizado los estudiantes residentes en las escuelas internadas, las personas ingresadas en hospitales ni las personas que han emigrado ni tampoco las familias nómadas y militares residentes en los territorios liberados.

Los saharauis sobreviven en la hammada donde sólo existen dos estaciones: El invierno que llega a $1 \mathrm{C}$, y en verano con temperaturas que rozan los 60 grados centígrados. En los Campamentos de refugiados saharauis, no se puede hablar de una economía propiamente dicha, ya que la principal actividad son el pastoreo y pequeños mercados de carácter privado que se ubican en cada municipio.

\section{El trabajo DE LA MUJER SAHARAUI EN SECTORES BÁSICOS}

\section{1. Ámbito cultural}

La mujer saharaui es la viva imagen de la identidad del pueblo saharaui con su inconfundible melhfa (vestimenta típica de las mujeres saharauis) de colores vivos, ella allá a donde va divulga la cultura y las tradiciones saharauis, sin olvidar que los hombres con su derraf (vestimenta típica saharaui importada de Mauritania) azul o blanco y su lzam (turbante), también hacen singular al pueblo saharaui

\section{2. Ámbito familiar}

La familia es el pilar fundamental de la sociedad saharaui. Las mujeres saharauis a la largo de muchos años han ejercido de madres, maestras y enfermeras durante los años del exilio. Actualmente las mujeres ocupan los mismos cargos y son las que se encargan del hogar y del cuidado de la familia. Las madres y abuelas tienen un papel fundamental en la cultura saharaui, como es transmitir a sus hijas los valores y tradiciones de la sociedad saharaui. 


\section{3. Ámbito educativo}

La educación en los campamentos de refugiados saharauis es uno de los campos prioritarios. Además, como dice el proverbio saharaui: "La educación es mejor que el origen".

Debido a la ausencia de los hombres por la lucha de la independencia del Sahara, las mujeres se convierten en las maestras al aire libre, ya que no existía las debidas infraestructuras y se escribía en tablas de madera o en la propia arena.

\section{4. Ámbito político}

Según un entrevistado, la mujer saharaui lleva incorporada en la política desde que el Sahara Occidental pasa a ser colonia española. A raíz de la colonización del Sáhara Occidental surgen movimientos anticolonistas y las mujeres participaban a favor de la independencia de su pueblo, según un entrevistado.

Así también lo reafirma Juliano D. (1998): "En los años 60, se produjo la formación inicial del Frente, en contra de España, hasta 1970 o 1973. Las mujeres se rebelaron todas contra el colonialismo español y fueron las primeras que se metieron en la lucha e hicieron cuadros, y respondieron al llamamiento que se había hecho de lucha en contra del colonialismo , y casi todas las células, en el inicio, en su mayoría estaban formadas por mujeres."

\section{5. Ámbito de la sanidad}

El ámbito de la sanidad es uno de los sectores más importantes y prioritarios para los saharauis, donde la mujer ha ejercido siempre con un papel ejemplar.

\section{PResentación y anÁlisis de los datos de entrevistas y Discusión}

\subsection{Entrevistas a saharauis residentes en los Campamentos de Refugiados saharauis}

a. La mayoría de los saharauis entrevistados residentes en los Campamentos, afirman que existe igualdad entre hombres y mujeres en oportunidades, derechos y obligaciones, y en los aspectos de formación y empleo.

Así lo expresan diferentes entrevistados: "La igualdad entre hombres y mujeres es el resultado de la necesidad del país a nivel nacional e internacional, cada vez que haya necesidad en alguno de los aspectos se orienta a mujeres y hombres".

b. Sin embargo, a pesar de que la mujer está presente en todas las instituciones y aspectos de la sociedad saharaui, su participación en la política es mínima, según los entrevistados, este hecho está justificados por las tradiciones y por la leyes de la sociedad que limitan la actividad de la mujeres en la política. 
Debido a este hecho, los saharauis ven que es imposible que una mujer se convierta en una figura política, como es acceder a la presidencia del Gobierno de la República Árabe Saharaui Democrática (RASD).

Consideran los entrevistados que en cuanto a toma de decisiones en el ámbito político, son los hombres, ya que la política es para hombres, ya que están más capacitados y así lo afirman algunos entrevistados:

"No tienen la misma capacidad los hombres que las mujeres, puesto que el hombre es mucho más consciente que la mujer. En cuanto a la toma de decisiones no consultan a las mujeres, ya que la política es para los hombres no para las mujeres".

Otro entrevistado afirma que: "En el trabajo político o actividades políticas, el hombre y la mujer no tienen las mismas capacidades para tomar las decisiones por las siguientes razones: El hombre tiene más experiencia que la mujer (guerra) y la mujer se inclina más a los sentimientos que a la razón".

c. La mayoría de los entrevistados consideran que la mujer saharaui ha hecho una gran labor en la construcción de la sociedad saharaui y la lucha por la independencia de su nación:

"La mujer ha jugado un papel muy importante durante la época colonial, como madre, educadora de las nuevas generaciones y enfermera. De una manera general ha jugado varios papeles en todo el proceso anterior y posterior a la guerra( administración, maestra, educadora...)".

"La mujer saharaui ha jugado un gran papel en la época colonial, organizó la lucha clandestina, ayudó en la reconstrucción del movimiento, recolectando dinero, armas y organizó manifestaciones".

d. En cuanto al ámbito familiar todos los entrevistados coinciden en que la mujer es la que se encarga del hogar y del cuidado de sus hijos. Los distintos entrevistados expresan su opinión a cerca de la mujer en este ámbito:

"En el aspecto familiar no hay comparación, la mujer es quién asume la responsabilidad en la educación de los hijos, ya que el hombre está en las zonas de combate y está ocupado la mayoría del tiempo".

e. En cuanto a los ámbitos de sanidad y enseñanza el número de mujeres es mayor debido las labores que han realizado las mujeres durante y después de la huida hacía el desierto.

Una entrevistada afirma que: " hay una gran cantidad de mujeres empleadas en el dominio de la salud, mucho más que los hombres, puesto que existe empleadas en este ámbito desde el año 1976 hasta la fecha".

Entre la diferencia o semejanza entre la mujer saharaui residente en los Campamentos de Refugiados Saharauis y la que reside en España; los saharauis entrevistados expresan:

"La mujer saharaui en los Campamentos y en España coinciden en los siguientes puntos: Político y de formación, y se diferencian en los aspectos cultural, sanitario y familiar". 
"Naturalmente que hay una gran diferencia entre la mujer saharaui en España y la que se encuentra en los Campos de refugiados, esta última está lejos de su realidad, incluso podría cambiar su forma de comportarse y adquirir otras mentalidades, lo cual contradice las costumbres y las tradiciones".

\subsection{Entrevistas a saharauis residentes en España}

a. La mayoría de los saharauis entrevistados creen que no existe una igualdad en cuanto a la formación:

"Igualdad real, desde luego que no. Igualdad de oportunidad, absoluta. Hilando más fino, si cabe, la igualdad de oportunidades es absoluta, porque todos los niños y niñas acceden a la escuela en idéntica igualdad de oportunidades. Lo que sucede, sucede después. Es decir, a las familias se les plantean problemas que, a su juicio, deben asumir las chicas. Por ejemplo, ante cuestiones como el cuidado de las personas mayores o la ayuda en casa o el cuidado de enfermos, las familias suelen optar por sacrificar a sus hijas antes que sacrificar a sus hijos".

b. La mayor parte de los entrevistados expresan que la mujer no participa en la política igual que los hombres:

"Político no, la mayoría son hombres y la decisión les corresponde a ellos. En el último congreso del Polisario, antes ni siquiera habían mujeres".

"Se ha hecho bastante, pero queda mucho por hacer. Por mucho que se quiere emancipar la mujer, pero no hay que olvidar que es una sociedad musulmana. Todo depende del nivel intelectual, y hay que formarse para llegar lejos".

c. Respecto al ámbito familiar los entrevistados en su conjunto piensan que la mujer es la que se encarga del hogar y de la responsabilidad familiar:

"En el ámbito familiar, la cuestión cambia un poco, en realidad es la mujer la que se ocupa de la mayor parte de los temas del hogar, al principio del conflicto era una situación forzada, ya que los hombres estaban en el Frente. Pero después ha pesado la idiosincrasia y las tradiciones, que concede ese papel a la mujer".

d. En relación a la sanidad y enseñanza los entrevistados argumentan que las mujeres a raíz del conflicto, ocupan puestos de trabajo relacionados con la educación (maestras) y la sanidad (enfermeras), ya que la mujer en los Campamentos de Refugiados Saharauis ha ejercido estas profesiones. Por lo que explican lo siguiente:

"En este caso, creo que sí hay igualdad, pero no en cuanto a los puestos de toma de decisiones dentro de la sanidad. No ha habido una ministra de sanidad, por ejemplo, los directores más importantes, hasta los sanitarios de las dairas son en su mayoría hombres".

e. De la misma forma los entrevistados expresan que la mujer ha hecho una gran labor durante la época colonial y los años posteriores: 
"Debido a las duras condiciones del conflicto y especialmente de los años de guerra, la mujer saharaui tuvo que tomar las riendas para sacar adelante a su pueblo. Ese papel que en principio fue "obligado" por la situación, actualmente es de lo más normal".

"La mujer ha perdido cotas de poder y de decisión a nivel familiar. Durante la época de la guerra, la mujer tenía mayores cotas de poder a nivel familiar y doméstico, por la sencilla razón de que no dependía del marido para obtener los medios de subsistencia".

\subsection{Entrevistas a asociaciones solidarias con el pueblo saharaui}

a. En cuanto a la opinión de los entrevistados españoles acerca de la mujer en los Campamentos de Refugiados Saharaui en el ámbito familiar, cultural, formación, política y salud, todos coinciden en que la mujer es el pilar fundamental del pueblo saharaui, así lo argumentan los entrevistados:

"En el ámbito familiar creo que es el pilar en torno al cual se mueve todo; en el cultural y de formación el grado de implicación en la educación como madres y maestras es incuestionable, en el político cada vez se va viendo más movimiento femenino a través de ocupación de cargos de importancia en distintos sectores, y en la salud su gran dedicación de profesionales sanitarias crece día a día".

b. En cuanto a si la mujer es libre para tomar sus decisiones, se expresan las siguientes opiniones donde la mayoría afirma que la mujer en gran medida depende del hombre:

"Pienso que desgraciadamente aún dependen mucho de la opinión de los varones de la unidad familiar. La igualdad no es plena y entiendo que la religión juega un papel muy importante en este sentido".

c. Un gran número de entrevistados considera que la mujer adquiere mayor autonomía e igualdad a raíz del conflicto de la independencia; respecto a esta cuestión comentan lo siguiente:

"Sin ninguna duda, la mujer saharaui en el conflicto se erige en protagonista fundamental, porque mientras los hombres estaban en el frente, ellas se ven como elementos que tienen que actuar porque sino no es posible nada, pero eso nunca hubiera sido posible sin una base, una personalidad dura a la vez que sensible .Porque es necesaria la firmeza y la sensibilidad para llevar a cabo lo que ellas llevaron. Lógicamente al adquirir unos valores nuevos y al llevarlos a la práctica era muy difícil que luego no fuesen legitimados, pero repito, siempre sobre una base y una predisposición de un pueblo."

e. La mayoría de los españoles afirman que la mujer indiscutiblemente es la promotora de los Campamentos de refugiados Saharauis:

"En parte sí, porque el papel que juega la mujer, se vio reforzado en la vida en los campamentos, ya que es donde comenzaron a ocupar puestos de 
protagonismo y dirección, y son las encargadas de la organización social y económica en los mismos".

\subsection{Comparación de la mujer saharaui con las mujeres musulmanas de los países vecinos como Argelia y Marruecos}

La mayoría de los entrevistados, tanto saharauis residentes en los Campamentos de Refugiados Saharauis como en España y los propios españoles, opinan que la mujer saharaui disfruta de unas condiciones y roles a nivel social y político que no es posible en los países vecinos. Así lo expresan algunos entrevistados:

"El maltrato a la mujer es motivo de repudio por parte del conjunto de la sociedad saharaui. La mujer saharaui goza de total autonomía para dar hospitalidad en su casa a toda persona aún en ausencia de cualquier miembro varón de la familia. En caso de divorcio tiene reconocido el derecho sobre la custodia de los hijos y el disfrute de los bienes familiares".

Así, otro entrevistado saharaui residente en los Campamentos de Refugiados Saharauis opina: "Eso es a consecuencia de la naturaleza misma de la sociedad, sus costumbres y sus tradiciones, se caracteriza por su independencia, siendo musulmana es diferente del resto de las mujeres de la región".

\section{CONCluSiones y PROPUESTAS}

La realización de esta investigación se ha llevado a cabo para analizar el papel de la mujer en la sociedad saharaui, dentro de un contexto muy difícil como es vivir en la hammada, ya que en muchas ocasiones a pesar de los muchos esfuerzos que se realizan no se ven reflejados los resultados. Aparte de las adversidades del exilio que tiene que superar la ciudadanía, a la mujer se le presentan otros obstáculos para seguir avanzando, como son las tradiciones, costumbres y la religión que de alguna forma hacen retroceder el avance de la mujer en el tiempo.

Al analizar la situación económica y social de los Campamentos de Refugiados saharauis, no se puede aplicar el concepto de desarrollo local sino un desarrollo a escala humana, ya que los saharauis dependen de la ayuda internacional por lo que no se puede tampoco hablar de una economía consolidada. Pero, a pesar de todas las circunstancias y los reveses que comporta el desierto y depender de la ayuda internacional, para poder sobrevivir, sobresale la capacidad de superación y de imaginación de la ciudadanía saharaui. En estos últimos años, los campamentos de refugiados saharauis han experimentado un notable desarrollo gracias a las ayudas provenientes de las familias españolas que acogen a los niños saharauis en el denominado Proyecto Vacaciones en Paz.

A lo largo del trabajo también se refleja que a pesar del papel fundamental que ha realizado la mujer en los Campamentos de Refugiados Saha- 
rauis, y la lucha por la independencia de su país, no ocupa altos cargos en la República Árabe Saharaui Democrática (RASD), ya que los hombres no la consideran preparada para ello o porque piensan que la política es cosa de hombres.

Por lo tanto creo, que para perseguir un desarrollo integrado para avanzar hay que contar con toda la ciudadanía y que si se excluye un grupo social de la sociedad ese desarrollo no es posible. Para la construcción de un estado, se tiene que contar con toda la sociedad y los distintos agentes para que formen parte de su desarrollo y puedan presentar buenas propuestas o prácticas que puedan mitigar las adversidades que presenta el exilio. Pero para llevar a cabo un estado participativo, es necesario un estado democrático, en este caso el gobierno saharaui lleva más de 36 años en el poder, por lo que se hace necesario una renovación del mismo para enfocar las incitativas del desarrollo de otra forma y así aportar nuevas ideas si se quiere un estado democrático.

En este caso es necesario debido a la situación de la población, contar con la ciudadanía para enfocar las ayudas o proyectos internacionales para satisfacer las necesidades básicas de la población. Por lo que se hace necesario implantar acciones y/o propuestas que favorezcan la igualdad entre hombres y mujeres , así como dictaminar leyes acerca de la equidad de participación entre mujeres y hombres y garantizar por parte del estado o los organismo locales que las mujeres puedan decidir libremente. La constitución de la República Árabe Saharaui Democrática no hace referencia a la participación de forma equitativa entre hombres y mujeres sino al derecho de participar en todos los ámbitos.

Como propuesta se para una mejor equidad y desarrollo se deben de implantar las siguientes acciones: Crecimiento de abajo hacia arriba, implantar acciones o buenas prácticas enfocadas a la igualdad entre mujeres y hombres, cursos o jornadas de sensibilización y concienciación a la sociedad a cerca de la capacidad de la mujer saharaui, cambio político y de viejas tradiciones para evolucionar hacia una sociedad abierta donde hay una igualdad real y equitativa entre mujeres y hombres.

\section{BIBLIOGRAFÍA, FUENTES BIBLIOGRÁFICAS Y ORALES}

BAJO ERRO, C. (2010): De la provincia 53 a la segunda gran traición. Testimonio de inmigrantes saharauis en España. Ed. ANARSD (Asociación Navarra de Amigos de la República Saharaui Democrática).

CÁNOVAS MARTÍ, LL. (2002): "Sáhara Occidental, la guerra olvidada", CLIO el pasado presente, n. 6, pp. 26-32.

DELCAMBRE, A.-M. (2006): Las Prohibiciones del Islam(Los derechos humanos, la Política, el Laicismo, la Mujer, el terrorismo). Ed. La esfera de los libros, Madrid. 
FABERO (2004): Proyecto DELEITE INMIGRACIÓN Y DESARROLLO LO$C A L$. Ed. UGT Castilla y León Secretaria de Acción Social. Castilla y Léon.

GONZÁLVEZ PÉREZ, V. (1994): "Descolonización y Migraciones desde el África Española (1956-1975)". Investigaciones Geográficas, no 12, Universidad de Alicante, Instituto Universitario de Geografía, pp. 45-84.

HIRSI ALI, A. (2006): Yo acuso (Defensa de la emancipación de las mujeres musulmanas), Traducción de Fernández Díaz, N. Ed. Galaxia Gutenberg, Círculo de Lectores. Madrid.

JULIANO, D. (1998): La Causa Saharaui y las Mujeres (siempre hemos sido muy libres). Ed. Icaria Más Madera, Barcelona.

Fuentes electrónicas:

http://www.mujeresaharauis.es

http://www.nubenegra.com/laMusicaHaul.html

http://www.cerocomasiete.es

Fuentes Orales:

Técnica de Cruz Roja Española

Esta información me la facilitan dos personas saharauis, un militar y un periodista, que trabajaban en el Ministerio de Información saharaui en la época de huida de los saharauis.

Diálogo durante las entrevistas con distintos saharauis.

Saharauis que han sido beneficiarios de las becas. 